\section{ORIG IN A L ARTICLE}

\title{
Cardiac microvascular rarefaction in hyperthyroid rats is reversed by losartan, diltiazem, and propranolol
}

\author{
Felipe Freitas, Vanessa Estato, Marcos A. Lessa, Eduardo Tibiriçá* \\ Laboratory of Cardiovascular Investigation, Oswaldo Cruz Institute, FIOCRUZ, Rio de Janeiro, Brazil
}

\section{Keywords}

capillary density, cardiac hypertrophy, cardiac microvascular rarefaction,

hyperthyroidism

Received 5 December 2013; revised 21 February 2014; accepted 27 March 2014

*Correspondence and reprints: etibi@ioc.fiocruz.br

\begin{abstract}
A B STRACT
Cardiac microvascular rarefaction appears to be involved in hyperthyroidisminduced left ventricular hypertrophy and dysfunction. We investigated the effects of losartan, an $\mathrm{AT}_{1}$ receptor antagonist; diltiazem, a calcium channel blocker; and propranolol, a $\beta$-adrenergic receptor antagonist, on cardiac function and structural microcirculatory cardiac alterations in an experimental model of L-thyroxininduced hyperthyroidism in rats. Hyperthyroidism (HYPER) was induced by intraperitoneal injections of L-thyroxin for 35 days $(600 \mu \mathrm{g} / \mathrm{kg} /$ day; $n=32)$. The euthyroid group was treated with distilled water (EUT + VEH; $n=8)$. On the 14 th day, the HYPER group was divided into four groups that received an oral treatment for 21 days with saline (HYPER + VEH; $n=8)$, losartan $(10 \mathrm{mg} / \mathrm{kg} /$ day; HYPER + LOS, $n=8)$, diltiazem $(10 \mathrm{mg} / \mathrm{kg} /$ day; HYPER + DILT, $n=8)$, or propranolol $(10 \mathrm{mg} / \mathrm{kg} /$ day; HYPER + PROP, $n=8)$. An echocardiographic study was performed at baseline, at the beginning and at the end of the pharmacological treatment protocol (35th day). The structural capillary density in the left ventricle (LV) was analyzed using histochemical analysis with fluorescein isothiocyanateconjugated Griffonia simplicifolia lectin. HYPER + VEH $(182 \pm 5 \mathrm{mmHg}$; $P<0.001)$ presented higher systolic blood pressure (SBP) compared with EUT + VEH $(132 \pm 3 \mathrm{mmHg})$. HYPER + LOS $(144 \pm 2 \mathrm{mmHg})$, HYPER + DILT $(147 \pm 3 \mathrm{mmHg})$ and HYPER + PROP $(153 \pm 4 \mathrm{mmHg})$ presented lower SBP compared with HYPER + VEH $(P<0.001)$. Chronic treatment with losartan, diltiazem, and propranolol reversed cardiac structural microvascular rarefaction

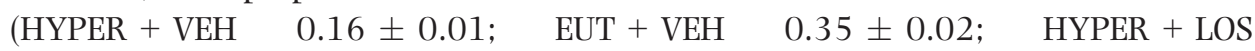
$0.46 \pm 0.03 ; \quad$ HYPER + DILT $0.49 \pm 0.02 ; \quad$ HYPER + PROP $0.58 \pm 0.04 \quad(\mathrm{Vv}$ [cap] $/ \mathrm{Vv}[\mathrm{fib}]) ; P<0.001)$ and enhanced the LV ejection fraction of hyperthyroid rats (HYPER + VEH $71 \pm 3 ; \quad$ EUT + VEH $85 \pm 2 ; \quad$ HYPER + LOS $90 \pm 3$; HYPER + DILT $85 \pm 3$; HYPER + PROP $86 \pm 2 \% ; P<0.05)$. In conclusion, chronic treatment with losartan, diltiazem, and propranolol improved the cardiac microcirculation and function in an experimental model of hyperthyroidism in rats.
\end{abstract}

\section{INTRODUCTION}

Hyperthyroid patients have a higher incidence of embolic events, stroke, ischemic heart disease, and congestive heart failure [1]. Although several studies have characterized the cardiovascular alterations of hyperthyroidism, cardiac microvascular dysfunction has not received as much attention [2-4].

It has been proposed that microvascular changes may play a substantial role in the development of cardiac failure in hypertensive patients [5]. Changes at the microvascular network level, such as arteriolar and 
capillary rarefaction, accompany many forms of hypertension in humans and animals [6,7]. In fact, several studies have emphasized the central involvement of the microcirculation in many cardiovascular diseases $[6,8,9]$.

Thus, it seems important to investigate microvascular structure and function to improve our knowledge of pathophysiological processes in many areas of cardiovascular disease, especially those involving hypertension, such as hyperthyroidism. Therefore, closer attention to the microcirculation may improve the treatment and prevention of cardiovascular events in hyperthyroidism. In this context, we recently showed, using an experimental model of hyperthyroidism in rats, that left ventricular (LV) dysfunction, cardiac hypertrophy, and myocardial fibrosis are associated with cardiac microvascular rarefaction [10]. Therefore, $\mathrm{LV}$ microcirculatory rarefaction may be involved in the pathophysiology of hyperthyroidism-induced cardiovascular alterations.

The sympathetic nervous system (SNS) and the renin-angiotensin system (RAS) have been implicated in the pathophysiology of cardiovascular changes induced by hyperthyroidism [11-14]. In fact, it is widely recognized that the alterations in hemodynamic parameters, including blood pressure and heart rate, resulting from enhanced SNS and RAS activity represent a central factor in cardiac hypertrophy induced by hyperthyroidism [14,15] and thus may alter cardiac microvascular function as well.

The SNS and RAS also induce cardiac remodeling in hyperthyroidism by altering intracellular calcium handling, suggesting that intracellular $\mathrm{Ca}^{2+}$ overload may play an important role in the myocardial hypertrophy induced by an excess of thyroid hormones [11,14,16]. In this context, it has been shown that $\mathrm{Ca}^{2+}$ channels have an important role in microvascular dysfunction in several animal models [17-20].

However, the effects of the SNS, the RAS, and calcium channel modulations on hyperthyroidism-induced microcirculatory rarefaction are not completely understood, and a better understanding of the role of these systems may contribute to a therapeutic strategy for cardiovascular alterations in hyperthyroid patients.

Thus, the main purpose of the present study was to investigate the effects of blockade of the RAS with losartan and the SNS with propranolol as well the effects of the calcium channel blocker diltiazem on hyperthyroidism-induced cardiac structural capillary rarefaction.

\section{MATERIALS AND METHODS}

\section{Animals}

Male Wistar rats weighing 170-250 g were used in the present study. During the course of the experiment, the animals were housed in Plexiglas cages under a 12-h light/dark cycle and received a diet of standard rat pellets and water ad libitum. All surgical procedures and protocols were approved by the Oswaldo Cruz Foundation Animal Welfare Committee (CEUA license \# L-034/08) and in accordance with the internationally accepted principles for the Care and Use of Laboratory Animals.

\section{Experimental protocol}

First, a rat model of hyperthyroidism was induced as previously described [21]. Briefly, intraperitoneal injections of L-thyroxin $\left(\mathrm{T}_{4}\right) \quad(600 \mu \mathrm{g} / \mathrm{kg} /$ day; diluted in $0.9 \% \mathrm{NaCl}$ ) were administered in the afternoon period (between 2 and $3 \mathrm{PM}$ ) for 14 consecutive days (HYPER; $n=32$ ). At the end of the 14th day, the HYPER group was divided into four groups that received, over the course of 21 days, an oral administration by gavage of saline (HYPER + VEH; $n=8$ ), losartan (10 mg/kg/day; HYPER + LOS, $n=8)$, diltiazem $(10 \mathrm{mg} / \mathrm{kg} /$ day; HYPER + DILT, $n=8)$, or propranolol (10 mg/kg/day; HYPER + PROP, $n=8)$. These hyperthyroid groups continued receiving daily intraperitoneal injections of $\mathrm{T}_{4}$ until the end of the experimental protocol, a total of 35 days. Animals in the control group received daily intraperitoneal injections of saline $0.9 \% \mathrm{NaCl}$ (EUT; $n=8$ ), and on the 14th day also started to receive distilled water by gavage for 21 days (EUT + VEH; $n=8$ ).

The hemodynamic and echocardiography evaluation was performed at baseline, on the 14th day and on the 35 th day. At the end of the $\mathrm{T}_{4}$ treatment protocol, the animals were anesthetized, and blood samples were collected from the inferior vena cava for measurement of the total triiodotironin $\left(\mathrm{T}_{3}\right)$ in the serum. After the blood collection, the animals were euthanized with an overdose of sodium pentobarbital, and the LV was immediately dissected, weighed, and placed in 4\% neutrally buffered paraformaldehyde for morphologic analysis.

\section{Hemodynamic measurements}

Systolic blood pressure (SBP) and heart rate (HR) were measured using a computerized tail-cuff plethysmography system (BP-2000; Visitech blood pressure analysis system, Apex, NC, USA) in conscious animals in the 
morning (8-12 AM). One week before the blood pressure measurement, the rats were familiarized with the prewarmed tail-cuff apparatus for three consecutive days to acclimatize them to the experimental procedure. SBP was measured at baseline, on the 14th day and at the end of the experimental protocol (35th day) in sessions of 5 min each with four animals per session.

\section{Echocardiographic measurements}

Echocardiographic analysis was performed in each animal at baseline, on the 14th day and at the end of the experimental protocol (35th day) under anesthesia (sodium pentobarbital, $50 \mathrm{mg} / \mathrm{kg}$, i.p.). A homeothermic blanket system was used to maintain normothermia (Harvard Apparatus, Boston, MA, USA). Echocardiography was performed with a commercially available ultrasound system (Titan ${ }^{\mathrm{TM}}$; SonoSite, Bothell, WA, USA) and a linear $10-\mathrm{MHz}$ high-frequency transducer. Care was taken to avoid excessive pressure on the thorax. The animals were placed in the left dorsal or lateral decubitus position. Parasternal short-axis views were obtained after the gain settings, and the angulation and rotation of the transducer were optimized to enable the imaging of both the epicardial and endocardial surfaces. Images were recorded at a frame rate of $200 \mathrm{~mm} / \mathrm{s}$, and twodimensional echocardiograms were obtained from shortaxis views of the $\mathrm{LV}$ at the level of the papillary muscles to measure and calculate thickness and ventricular diameters. The investigator was blinded to the experimental group, and all measurements were made from the digital images captured at the time of the study.

The LV end-diastolic dimension, LV posterior wall end-diastolic thickness, interventricular septal thickness, LV fractional shortening (FS), and LV ejection fraction were measured according to the recommendations of the American Society of Echocardiography for 2D echocardiography [22]. To determine the echocardiographic LV mass, we used the standard cube function formula: $\mathrm{LV}$ mass $(\mathrm{mg})=1.04 \times\left[(\mathrm{IVS}+\mathrm{EDD}+\mathrm{PWT})^{3}-(\mathrm{EDD})^{3}\right]$, where 1.04 is the specific gravity of muscle [23], IVS is the end-diastolic interventricular septal thickness, EDD is the LV end-diastolic dimension, and PWT is the enddiastolic posterior wall thickness.

\section{Serum hormone measurements}

Serum concentrations of $\mathrm{T}_{3}$ were measured using an electrochemiluminescence immunoassay kit (Roche Molecular Biochemical, Indianapolis, IN, USA). This methodology has been frequently used in the assessment of total serum levels of $\mathrm{T}_{3}[24,25]$.

\section{Structural capillary density}

The LV samples were dehydrated in a graded series of ethanol $(70,95$, and $100 \%)$ and embedded in paraffin. The left ventricular structural capillary density was determined as previously described [26]. Briefly, this method generates isotropic, uniform, and random sections of biological specimens for the quantitative assessment of three-dimensional anisotropic structures from two-dimensional sections. Although the myocardium is an anisotropic structure, isotropic sections are required for stereological studies. Therefore, the organ was cut using the 'orthrip' method [27], which creates uniform isotropic sections by consecutively dividing the fragment three times. The first section is random, the second section is orthogonal to the first, and the third section is orthogonal to the second. The paraffin blocks were cut into 5 - $\mu \mathrm{m}$-thick sections and stained with FITC-conjugated Griffonia simplicifolia I lectin (1: 150 dilution) in a dark, humidified chamber at room temperature for $30 \mathrm{~min}$. For each rat, at least seven randomly selected microscopic fields were examined for three different tissue sections. The volume density of the capillaries $(\mathrm{Vv}[\mathrm{cap}])$ was calculated as follows: $\mathrm{Vv}$ [cap] $=\mathrm{Pp} / \mathrm{PT}(\%)$, where Pp is the number of points coincident with a capillary and PT is the total number of test points ( $\mathrm{PT}=56$ in the present case). The fiber volume density ( $\mathrm{Vv}[\mathrm{fib}])$ was also calculated. The capillary volume density divided by fiber volume density $(\mathrm{Vv}[\mathrm{cap}] / \mathrm{Vv}[\mathrm{fib}])$ was calculated to overcome any influence of cardiac hypertrophy on the myocardial capillary density.

\section{Assessment of cardiac fibrosis by picrosirius red staining}

The LV was transversely sectioned one time at the midpoint between apex and base, and a cross-sectional slice from each sample was prepared and embedded in paraffin wax [2]. Five-micrometer-thick sections were cut, mounted onto glass slides, and stained with picrosirius red. For each sample, at least ten randomly selected microscopic fields from the LV were captured at a magnification of $200 \times$ (Olympus BX50 and Olympus UC30 scanning unit; Olympus, Center Valley, PA, USA), and the $\%$ area of collagen content was assessed using Image Pro-Plus ${ }^{\circledR}$ software version 6.2 (Media Cybernetics Inc., Bethesda, MD, USA). To determine the $\%$ area of collagen deposition, we used the standard formula: $\mathrm{LV}$ collagen deposition $(\%)=\mathrm{CA} \times 100 / \mathrm{TA}$, where CA is the collagen area of each microscopic field and TA is the total area of the microscopic field. 


\section{Statistical analyses}

The results were expressed as the mean \pm standard error of the mean (SEM) for each group, and betweengroup comparisons were made by analysis of variance (ANOVA). When significant differences were detected by ANOVA, the Bonferroni test was used to find statistically significant differences. Differences with $P$ values $<0.05$ were considered significant. All calculations were performed by computerized analysis using commercially available statistical programs (Graphpad Instat and Graphpad Prism, Graphpad Software, Inc., La Jolla, CA, USA).

\section{Drugs}

All drugs used were purchased from Sigma Chemical Co., St. Louis, MO, USA, including sodium pentobarbital, L-thyroxin, losartan, diltiazem, and propranolol.

\section{RES ULTS}

\section{Body weight, echocardiographic, and hemodynamic parameters on 14th day in hyperthyroid rats}

There were no significant differences in the basal values of body weight between EUT and HYPER groups (Table I). At the baseline evaluation, SBP, HR, and echocardiography parameters were not different among the different experimental groups (data not shown).

The HYPER group showed LV hypertrophy, with a marked increase in PWT and IVS $(P<0.001)$ compared with the EUT group. The LV ejection fraction was significantly reduced in the HYPER group compared with the EUT group $(P<0.05)$. Finally, EDD and FS were not modified in hyperthyroid rats (Table I).

L-thyroxin administration resulted in increases in SBP and HR in the HYPER group compared with the EUT group $(P<0.001)$. In the HYPER group, body weight $(14$ th day) $(P<0.01)$ was decreased, whereas $\mathrm{LV}$ and heart mass were markedly increased, when compared with the EUT group $(P<0.001$; Table I).

\section{Effects of chronic treatment with losartan, diltiazem, and propranolol on body weight, serum total triiodothyronine level, and hemodynamic and echocardiographic parameters on the 35th day in hyperthyroid rats}

Body weight was decreased in all hyperthyroid groups when compared with the EUT + VEH group, and the treatment with losartan, diltiazem, or propranolol did not modify body weight when compared with the
Table I Body weight, hemodynamic, and echocardiographic parameters on the 14th day in hyperthyroid rats before treatment with losartan, diltiazem, and propranolol.

\begin{tabular}{lcc}
\hline Parameters & EUT $(n=8)$ & HYPER $(n=32)$ \\
\hline BW day 0 (g) & $284 \pm 13$ & $280 \pm 5$ \\
BW day 14 (g) & $317 \pm 10$ & $288 \pm 3^{* *}$ \\
SBP (mmHg) & $140 \pm 4$ & $183 \pm 1^{* * *}$ \\
HR (bpm) & $358 \pm 10$ & $438 \pm 7^{* * *}$ \\
Heart mass (g) & $0.97 \pm 0.01$ & $1.16 \pm 0.02^{* * *}$ \\
LV mass (g) & $0.46 \pm 0.02$ & $0.65 \pm 0.02^{* * *}$ \\
PWT (mm) & $1.5 \pm 0.1$ & $2.1 \pm 0.1^{* * *}$ \\
IVS (mm) & $1.4 \pm 0.1$ & $1.9 \pm 0.1^{* * *}$ \\
EDD (mm) & $5.5 \pm 0.3$ & $5.4 \pm 0.1$ \\
FS (\%) & $47 \pm 1$ & $51 \pm 1$ \\
LV EF $(\%)$ & $85 \pm 1$ & $75 \pm 3^{*}$ \\
\hline
\end{tabular}

L-thyroxin treatment: $600 \mu \mathrm{g} / \mathrm{kg} /$ day i.p. for 14 days (HYPER). SBP, systolic blood pressure; HR, heart rate; BW, body weight; EDD, left ventricular (LV) end-diastolic dimension; FS, LV fractional shortening; EF, ejection fraction; IVS, end-diastolic interventricular septal thickness; PWT, end-diastolic posterior wall thickness. Animals in the control group received daily intraperitoneal injections of saline $0.9 \% \mathrm{NaCl}$ (EUT).

Values represent the means \pm SEM

$* P<0.05, * * P<0.01$ and $* * * P<0.001$ vs. euthyroid rats.

HYPER + VEH group (Table II). HYPER + VEH presented significantly elevated SBP $(P<0.001)$ and HR $(P<0.01)$ compared with euthyroid rats. The treatments with losartan, diltiazem, or propranolol for 21 days were able to reduce SBP in hyperthyroid rats $(P<0.001)$. In contrast, when compared with HYPER + VEH, HR was reduced only after propranolol treatment $(P<0.05$; Table II). L-thyroxin administration resulted in a marked increase of serum total $\mathrm{T}_{3}$ in all hyperthyroid groups compared with euthyroid rats that received saline injections for the same period $\left(P<0.001\right.$; Table II). Serum total $\mathrm{T}_{3}$ did not differ in hyperthyroid rats treated with losartan, diltiazem, or propranolol when compared with the HYPER + VEH group (Table II).

Table II shows that HYPER + VEH exhibited cardiac hypertrophy, with a marked increase in heart and LV mass $(P<0.01)$, PWT and IVS $(P<0.001)$ and a reduced EDD $(P<0.05)$ compared with EUT + VEH. Non-treated hyperthyroid rats presented a significantly reduced LV ejection fraction compared with euthyroid rats $(P<0.05)$. The treatment of hyperthyroid rats with losartan, diltiazem, or propranolol was able to reverse cardiac hypertrophy and restore LV EDD and the ejection fraction in the echocardiographic assessment, as shown in Table II. FS was not modified in any group compared with euthyroid rats. 
Table II Body weight, serum total triiodothyronine level, hemodynamic, and echocardiographic parameters in hyperthyroid rats after chronic treatment with losartan, diltiazem, and propranolol

\begin{tabular}{|c|c|c|c|c|c|}
\hline Parameters & EUT + VEH & HYPER + VEH & HYPER + LOS & HYPER + DILT & HYPER + PROP \\
\hline BW day 35 (g) & $335 \pm 12$ & $294 \pm 8^{*}$ & $279 \pm 4 * *$ & $290 \pm 12^{*}$ & $300 \pm 3^{*}$ \\
\hline Total $T_{3}$ (ng/dL) & $106 \pm 3$ & $319 \pm 20^{* * *}$ & $295 \pm 23^{* * *}$ & $308 \pm 27^{* * *}$ & $299 \pm 14^{* * *}$ \\
\hline $\mathrm{SBP}(\mathrm{mmHg})$ & $132 \pm 3$ & $182 \pm 5^{* * *}$ & $144 \pm 2^{\# \# \#}$ & $147 \pm 3^{\# \# \#}$ & $153 \pm 4^{\# \# \#}$ \\
\hline $\mathrm{HR}$ (bpm) & $446 \pm 2$ & $533 \pm 21^{* *}$ & $530 \pm 22 *$ & $499 \pm 16^{*}$ & $462 \pm 6^{\#}$ \\
\hline Heart mass (g) & $1.0 \pm 0.02$ & $1.3 \pm 0.03^{* *}$ & $1.1 \pm 0.03^{\#}$ & $1.0 \pm 0.04^{\#}$ & $1.1 \pm 0.06^{\#}$ \\
\hline LV mass $(\mathrm{g})$ & $0.54 \pm 0.02$ & $0.75 \pm 0.06^{* *}$ & $0.60 \pm 0.02^{\#}$ & $0.59 \pm 0.03^{\#}$ & $0.57 \pm 0.03^{\#}$ \\
\hline PWT (mm) & $1.7 \pm 0.06$ & $2.3 \pm 0.06 * * *$ & $1.9 \pm 0.09^{\# \#}$ & $1.8 \pm 0.06^{\# \#}$ & $1.7 \pm 0.13^{\# \#}$ \\
\hline IVS (mm) & $1.4 \pm 0.06$ & $2.1 \pm 0.03^{* * *}$ & $1.6 \pm 0.07^{\# \# \#}$ & $1.7 \pm 0.04^{\# \#}$ & $1.5 \pm 0.06^{\# \# \#}$ \\
\hline $\mathrm{EDD}(\mathrm{mm})$ & $5.7 \pm 0.1$ & $4.9 \pm 0.1^{*}$ & $5.8 \pm 0.2^{\#}$ & $5.8 \pm 0.4^{\#}$ & $5.9 \pm 0.1^{\#}$ \\
\hline FS (\%) & $50 \pm 3$ & $50 \pm 5$ & $58 \pm 5$ & $49 \pm 3$ & $51 \pm 4$ \\
\hline LV EF (\%) & $85 \pm 2$ & $71 \pm 3^{*}$ & $90 \pm 3^{\# \#}$ & $85 \pm 3^{\#}$ & $86 \pm 2^{\#}$ \\
\hline
\end{tabular}

Intraperitoneal injections of L-thyroxin $(600 \mu \mathrm{g} / \mathrm{kg} / \mathrm{day}$; diluted in $0.9 \% \mathrm{NaCl})$ were administered for 14 consecutive days. At the end of the 14 th day, the hyperthyroid rats were treated orally for 21 days with saline (HYPER + VEH; $n=8)$, losartan (10 mg/kg/day; HYPER + LOS, $n=8)$, diltiazem (10 mg/kg/day; HYPER + DILT, $n=8)$, or propranolol (10 mg/kg/day; HYPER + PROP, $n=8)$. The hyperthyroid groups continued receiving daily intraperitoneal injections of $\mathrm{T}_{4}$ until the end of the experimental protocol (35th day). The control group received daily intraperitoneal injections of saline $0.9 \% \mathrm{NaCl}$ and on the 14 th day also started to receive distilled water by gavage for 21 days (EUT + VEH; $n=8$ ). SBP, systolic blood pressure; HR, heart rate; BW, body weight; $T_{3}$, triiodothyronine; EDD, left ventricular (LV) end-diastolic dimension; FS, LV fractional shortening; EF, ejection fraction; IVS, end-diastolic interventricular septal thickness; PWT, end-diastolic posterior wall thickness.

Values are the means \pm SEM.

$* P<0.05, * * P<0.01,{ }^{* * * P}<0.001$ Vs. EUT + VEH; ${ }^{\#} P<0.05,{ }^{\# \#} P<0.01,{ }^{\# \#} P<0.001$ Vs. HYPER + VEH.

\section{Effects of chronic treatment with losartan, diltiazem, and propranolol on the left ventricular structural capillary density of hyperthyroid rats}

Hyperthyroid rats presented with myocardial capillary rarefaction characterized by a $54 \%$ reduction in the $\mathrm{LV}$ structural capillary density compared with euthyroid rats (HYPER + VEH $0.16 \pm 0.01$ vs. EUT + VEH $0.35 \pm 0.02$ capillary volume density-to-fiber volume density ratio $(\mathrm{Vv}[\mathrm{cap}] / \mathrm{Vv}[\mathrm{fib}]) ; P<0.001)$, as shown in Figure $1 a, b$ and $f$. Hyperthyroid rats treated with losartan (HYPER + LOS $0.46 \pm 0.03 \mathrm{Vv}[\mathrm{cap}] / \mathrm{Vv}[\mathrm{fib}]$; $P<0.001$ ), diltiazem (HYPER + DILT $0.49 \pm 0.02 \mathrm{Vv}$ [cap]/Vv[fib]; $P<0.001$ ), or propranolol (HYPER + PROP $0.58 \pm 0.04 \mathrm{Vv}[\mathrm{cap}] / \mathrm{Vv}[\mathrm{fib}] ; P<0.001)$ exhibited a marked increase of 86,95 , and $120 \%$, respectively, in LV structural capillary density when compared with non-treated hyperthyroid rats (HYPER + VEH $\quad 0.16 \pm 0.01 \quad \mathrm{Vv}[\mathrm{cap}] / \mathrm{Vv}[\mathrm{fib}]), \quad$ as shown in Figure $1 c-f$.

\section{Effects of chronic treatment with losartan, diltiazem, and propranolol on left ventricle collagen deposition of hyperthyroid rats}

In the picrosirius red-stained sections, we observed extensive areas of collagen deposition in the LV of the non-treated hyperthyroid rats compared with the euthyroid rats (HYPER + VEH $9.8 \pm 1.3 \%$ vs. EUT + VEH $4.3 \pm 0.4 \% ; \quad P<0.001)$, as shown in Figure $2 a, b$ and $f$. The treatment of hyperthyroid rats with losartan $(3.6 \pm 0.4 \% ; \quad P<0.001)$, diltiazem $(4.7 \pm 0.7 \% ; P<0.001)$, or propranolol $(3.2 \pm 0.2 \%$; $P<0.001)$ significantly reduced the percentage of fibrosis areas in the LV compared with non-treated hyperthyroid rats, as shown in Figure $2 c-f$.

\section{DISCUSSION}

The main findings of the present study are that chronic treatment with losartan, diltiazem, and propranolol (i) reverse cardiac structural microvascular rarefaction, (ii) reduce cardiac hypertrophy, (iii) enhance LV function, and (iv) reduce collagen deposition in the LV of hyperthyroid rats. We also demonstrated that chronic thyroxin administration produces clinical, biochemical, and pathological evidence of hyperthyroidism, including tachycardia, systolic hypertension, body weight loss, and increased serum levels of total $\mathrm{T}_{3}$.

We recently demonstrated that $\mathrm{T} 4$ treatment $(600 \mu \mathrm{g} / \mathrm{kg} / \mathrm{day}$, ip during 14 days) induces a hyperthyroidism state characterized by LV dysfunction accompanied by cardiac microvascular rarefaction [10]. The influence of SNS and RAS on the cardiovascular 

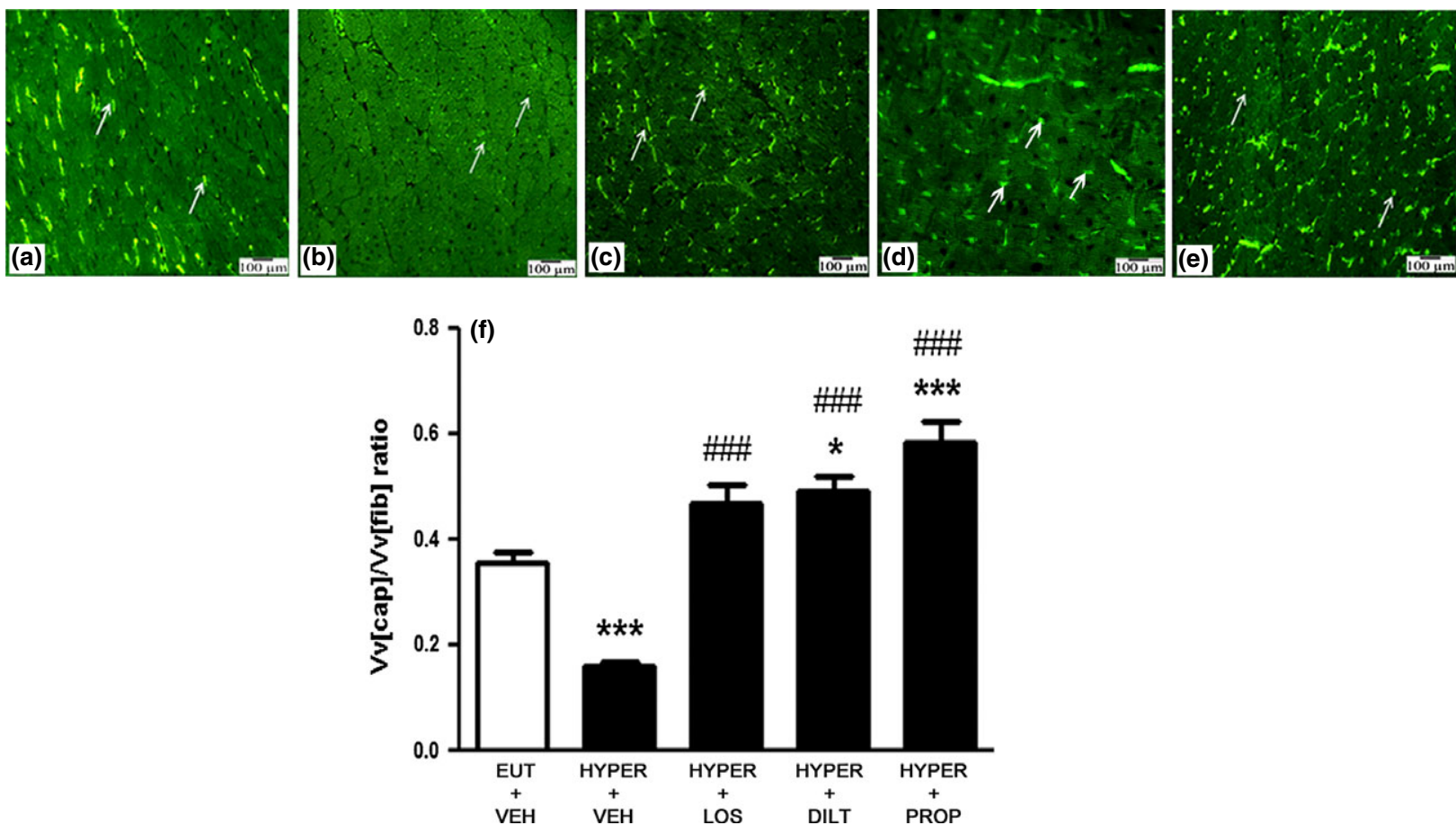

Figure 1 Representative photomicrographs of left ventricle (LV) sections: FITC-conjugated Griffonia simplicifolia lectin staining of capillaries in (a) euthyroid animals that received distilled water by gavage for 21 days (EUT + VEH; $n=8$ ), (b) hyperthyroid rats treated with saline $0.9 \% \mathrm{NaCl}$ (HYPER + VEH), (c) hyperthyroid rats treated with losartan $(10 \mathrm{mg} / \mathrm{kg} /$ day; HYPER $+\mathrm{LOS}, n=8)$,

(d) hyperthyroid rats treated with diltiazem $(10 \mathrm{mg} / \mathrm{kg} /$ day; HYPER + DILT, $n=8)$, and (e) hyperthyroid rats treated with propranolol $(10 \mathrm{mg} / \mathrm{kg} /$ day; HYPER + PROP, $n=8)$. Quantitative analysis of the LV capillary volume density-to-fiber volume density ratio (f). Values are the means \pm SEM. ${ }^{*} P<0.05,{ }^{* * *} P<0.001$ vs. EUT + VEH; ${ }^{\# \#} P<0.001$ vs. HYPER + VEH.

alterations present in hyperthyroidism is well characterized. However, no previous studies have investigated the effects of losartan, diltiazem, and propranolol on microcirculatory alterations in hyperthyroidism models. In this context, this is the first study to demonstrate that chronic treatment with losartan, an $\mathrm{AT}_{1}$ receptor blocker; diltiazem, a calcium channel antagonist; and propranolol, a $\beta$-adrenoceptor antagonist is able to reverse cardiac microvascular rarefaction in experimental hyperthyroidism. We have previously shown that RAS inhibition reverses the cardiac structural capillary rarefaction in different models of hypertension, such as spontaneously hypertensive rats and in diabetic hypertensive rats $[26,28]$. Calcium channel blockers have been shown to increase microvessel density in hypertension models [18,19]. However, $\beta$-adrenergic receptor antagonists not always reverse myocardial capillary rarefaction in hypertension [7]. In this context, due to the increase in myocardial $\beta$-adrenergic responsiveness in hyperthyroidism [13], the $\beta$-adrenergic receptor blockade in our study was able to markedly increase left ventricular structural capillary density in hyperthyroid rats.

It is widely recognized that the augmented hemodynamic parameters resulting from higher SNS and RAS activity represent a central factor in the cardiac remodeling in hyperthyroidism [15]. Moreover, the SNS and RAS are involved in microvascular rarefaction in several cardiovascular diseases and experimental models of hypertension, diabetes, and metabolic syndrome [28-31]. In addition, systemic microvascular rarefaction might be a primary cardiovascular alteration in essential hypertension [7]. In contrast, the structural capillary rarefaction observed in our study may represent a downstream consequence of secondary hypertension and cardiac hypertrophy in hyperthyroidism.

In hypertension, the mechanisms regulating vasomotor tone are altered, leading to enhanced vasoconstriction or reduced vasodilator responses. Anatomic alterations in the structure of individual precapillary resistance vessels, such as an increase in their wall-tolumen ratio [7], may result in the temporary obstruction 

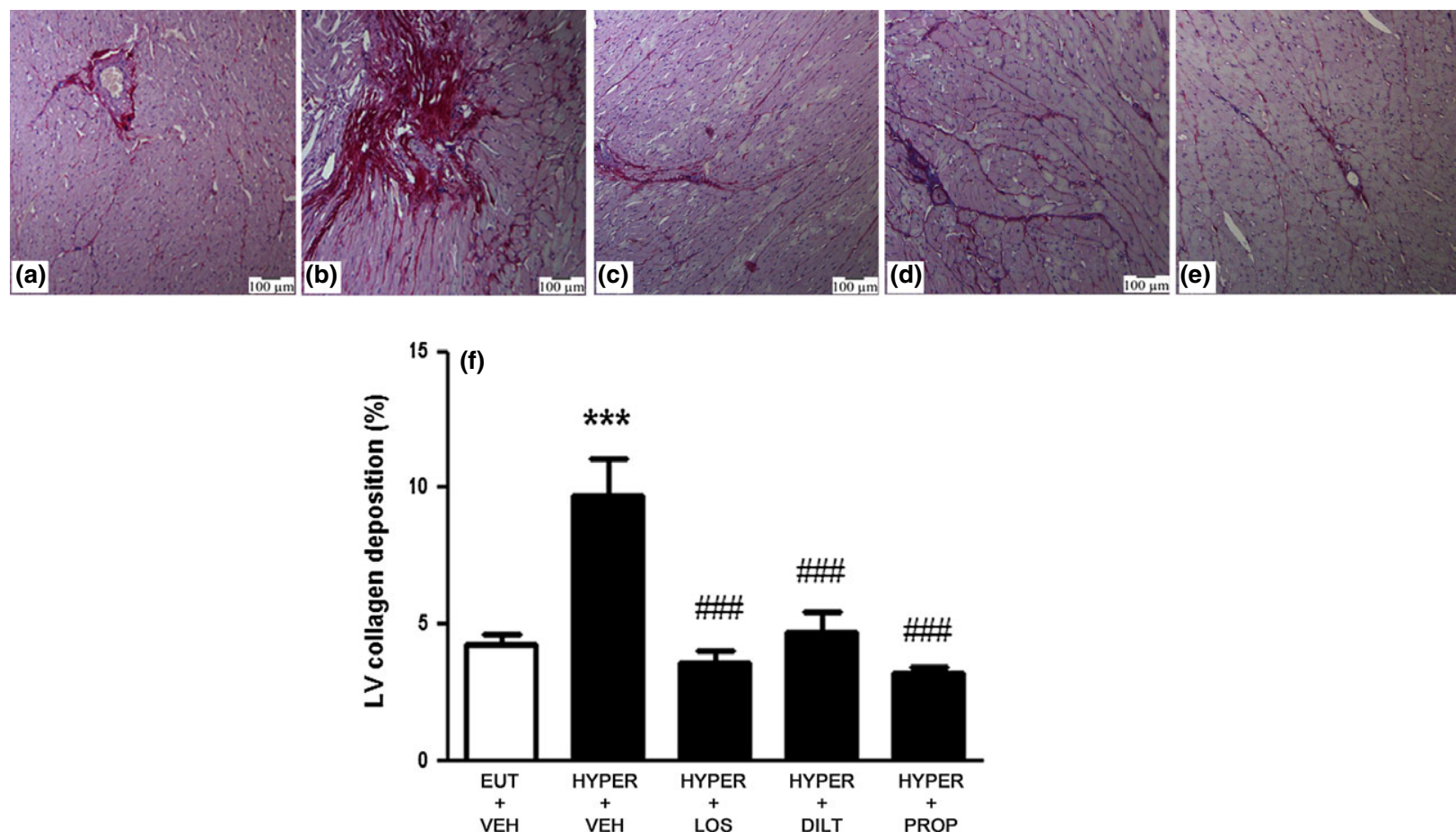

Figure 2 Representative photomicrographs of left ventricle (LV) sections: Picrosirius red staining for the evaluation of fibrosis in (a) euthyroid animals that received distilled water by gavage for 21 days (EUT + VEH; $n=8$ ), (b) hyperthyroid rats treated with saline $0.9 \%$ $\mathrm{NaCl}($ HYPER + VEH), (c) hyperthyroid rats treated with losartan $(10 \mathrm{mg} / \mathrm{kg} /$ day; HYPER $+\mathrm{LOS}, n=8)$, (d) hyperthyroid rats treated with diltiazem (10 mg/kg/day; HYPER + DILT, $n=8)$, and (e) hyperthyroid rats treated with propranolol $(10 \mathrm{mg} / \mathrm{kg} / \mathrm{day}$; HYPER + PROP, $n=8$ ). Quantitative analysis of the LV collagen deposition (f). Values are the means \pm SEM. $* * * P<0.001$ vs. EUT + VEH; ${ }^{\# \# \# P}<0.001$ vs. HYPER + VEH.

of blood flow (functional capillary rarefaction) followed by a disappearance of the capillaries (structural capillary rarefaction) by apoptosis [32]. Endothelial cell apoptosis plays a critical role in the disruption of microcirculation. The apoptosis of a relatively small proportion of endothelial cells may be sufficient to mediate significant microvessel rarefaction [33].

In the present work, we demonstrated that 21-day treatment with losartan, diltiazem, and propranolol significantly reduced SBP in hyperthyroid rats. We have previously shown that antihypertensive drugs from different pharmacological classes have distinct abilities for reversing functional and/or structural microvascular alterations in arterial hypertension [26]. In the present study, using an experimental model of hyperthyroidism-induced secondary hypertension, we showed that different pharmacological classes of antihypertensive drugs have similar effects on blood pressure that are accompanied by a reversion of LV structural capillary rarefaction. It is important to note that improvement in capillary density can be attributed to blood pressure reduction itself in normotensive and hypertensive subjects [34]. Blood pressure reduction per se is accompanied by a reduction in biomechanical and oxidative stress [35] that can result in improvements of microvascular function. Therefore, the lower SBP observed in hyperthyroid rats treated with the drugs investigated in the present study may be involved in the reversal of cardiac microcirculatory rarefaction. Similarly, it has been described in clinical and experimental studies that long-term antihypertensive treatment with drugs from different pharmacological classes results in the normalization of capillary density, and, in some cases, the capillary density of treated individuals is even superior to that of control subjects $[28,36,37]$.

The reduced myocardial capillary density observed in our study may reflect a failure of microcirculatory growth (angiogenesis) that would compensate the progressive increase in myocardial mass and cardiomyocyte dimensions. These two processes are highly influenced by the upregulation of RAS and SNS present in hyperthyroidism [38]. In fact, the treatment with 
losartan, diltiazem, and propranolol also reversed cardiac hypertrophy, prevented the establishment of myocardial fibrosis, and restored LV function in hyperthyroid rats. In this context, it has described that improvement of LV function induced by $\beta$-blockade is also associated to the preservation of myocardial highenergy phosphate intracellular levels in patients with heart failure [39]. This pharmacological effect of $\beta$ blockers could also be involved in the enhancement of LV function in hyperthyroid rats. Finally, the reversal of cardiac structural microvascular rarefaction in hyperthyroid rats submitted to chronic treatment with losartan, diltiazem, and propranolol may be explained by the protective effect of these drugs on cardiac remodeling.

The improvement in LV structural capillary density demonstrated in our study by the elevated LV capillary/fiber ratio might also explain the beneficial cardiac effects of RAS and SNS blockade observed in hyperthyroidism. Microvascular rarefaction, especially in highly perfused organs such as the heart, reduces oxygen supply and the exchange of metabolic substrates, which in turn might contribute to organ dysfunction. Therefore, the LV dysfunction observed in the hyperthyroid rats in our study may be related to the combination of reduced myocardial capillary density, arterial hypertension, and cardiac fibrosis. The improvement in cardiac capillary density observed in hyperthyroid rats treated with losartan, diltiazem, and propranolol may have enhanced myocardial perfusion, thereby preventing tissue hypoxia, cardiac fibrosis, and LV dysfunction.

It was previously demonstrated that both the SNS and RAS can induce cardiac remodeling in hyperthyroidism by inducing intracellular $\mathrm{Ca}^{2+}$ overload, which may have an important role in the cardiovascular alterations induced by the excess of thyroid hormones [11,14,16]. Additionally, Wu et al. [16] showed that regulatory proteins involved in intracellular calcium cycling, including cardiac ryanodine receptor 2 (RyR2) and sarcoplasmic reticulum $\mathrm{Ca}^{2+}$-ATPase (SERCA) mRNA, were overexpressed in the hearts of hyperthyroid rats. In addition, it has been suggested that the upregulation of $\mathrm{Ca}^{2+}$ channels is associated with both LV hypertrophy and diastolic heart failure in other experimental models [40,41]. Su et al. [11] showed that $\mathrm{AT}_{1}$ receptor blockade has beneficial effects on hyperthyroid myocardial hypertrophy by altering intracellular calcium handling. In addition, Wu et al. [16] reported that treatment with propranolol and verapamil, a calcium channel antagonist, inhibited both
RyR2 and SERCA mRNA over-expression and prevented cardiovascular alterations in hyperthyroid rats. However, the positive microcirculatory effects induced by the blockade of the $\mathrm{AT}_{1}$ and the $\beta$-adrenergic receptors, as well as of the calcium channels, as observed in our study, have never been described. Our data are in agreement with other studies that showed, in different experimental models, that calcium channel blockers have a pro-angiogenic effect in the heart [17,42]. In fact, calcium antagonists have been shown to increase microvessel density in several animal models [18-20].

Therefore, the regulation of intracellular calcium handling and the modulation of $\mathrm{Ca}^{2+}$ channels may underlie the induction of angiogenesis observed after treatment with losartan, propranolol, and diltiazem in our experimental model of hyperthyroidism. These factors may also explain the positive effects of the diltiazem treatment, which, in addition to inhibiting apoptosis, induces angiogenesis in the myocardial microcirculation.

Clinically, hyperthyroidism influences hemodynamics and can induce coronary vasospasm [43-47]. Even if the mechanisms responsible for vasospasm in hyperthyroidism remain unknown, angina, myocardial ischemia, and infarction in hyperthyroid patients usually occur in conjunction with preexisting coronary artery disease due to a supply-and-demand imbalance [4347]. Nevertheless, in many clinical cases, the use of calcium channel blockers is recommended in the treatment of coronary spasm induced by hyperthyroidism [43-47], suggesting that calcium metabolism might be directly associated with the coronary microvascular dysfunction induced by hyperthyroidism. It has also been suggested that microvascular changes may make a substantial contribution to the development of cardiac failure in hypertensive patients [5]. Hence, structural changes of the myocardium, such as microcirculatory rarefaction, may have profound effects on the performance of the LV and on the long-term prognosis of hyperthyroidism.

Finally, losartan, propranolol, and diltiazem could be used as an adjuvant therapy in the treatment of hyperthyroidism considering their beneficial microcirculatory effects and their ability to prevent cardiac capillary rarefaction resulting from chronic hyperthyroidism.

\section{DECLARATION OF INTEREST}

No conflicts of interest, financial or otherwise, are declared by the authors.

C 2014 Société Française de Pharmacologie et de Thérapeutique Fundamental \& Clinical Pharmacology 29 (2015) 31-40 


\section{FUNDING}

This work was supported by grants from CNPq (Conselho Nacional de Desenvolvimento Científico e Tecnológico), FAPERJ (Fundação de Amparo à Pesquisa do Estado do Rio de Janeiro), and the Oswaldo Cruz Foundation (Fiocruz).

\section{AUTHOR CONTRIBUTION}

E.T. and M.A.L conceived and design the research; F.F and V.E. performed the experiments; E.T., F.F, V.E., and M.A.L analyzed data and interpreted the results of the experiments; F.F. drafted the manuscript; E.T., V.E., and M.A.L edited and revised the manuscript; and E.T. approved the final version of the manuscript.

\section{REFERENCES}

1 Siu C.W., Yeung C.Y., Lau C.P., Kung A.W., Tse H.F. Incidence, clinical characteristics and outcome of congestive heart failure as the initial presentation in patients with primary hyperthyroidism. Heart (2007) 93 483-487.

2 Anjos-Ramos L., Carneiro-Ramos M.S., Diniz G.P., MartinsSilva J., Barreto-Chaves M.L. Early cardiac hypertrophy induced by thyroxine is accompanied by an increase in VEGFA expression but not by an increase in capillary density. Virchows Arch. (2006) 448 472-479.

3 Heron M.I., Kolar F., Papousek F., Rakusan K. Early and late effect of neonatal hypo- and hyperthyroidism on coronary capillary geometry and long-term heart function in rat. Cardiovasc. Res. (1997) 33 230-240.

4 Tomanek R.J., Connell P.M., Butters C.A., Torry R.J. Compensated coronary microvascular growth in senescent rats with thyroxine-induced cardiac hypertrophy. Am. J. Physiol. (1995) 268 419-425.

5 Strauer B.E. Significance of coronary circulation in hypertensive heart disease for development and prevention of heart failure. Am. J. Cardiol. (1990) 65 34G-41G.

6 Struijker-Boudier H.A., Rosei A.E., Bruneval P. et al. Evaluation of the microcirculation in hypertension and cardiovascular disease. Eur. Heart J. (2007) 28 2834-2840.

7 Levy B.I., Ambrosio G., Pries A.R., Struijker-Boudier H.A. Microcirculation in hypertension: a new target for treatment? Circulation (2001) 104 735-740.

8 Debbabi H., Bonnin P., Levy B.I. Effects of blood pressure control with perindopril/indapamide on the microcirculation in hypertensive patients. Am. J. Hypertens. (2010) 231136 1143.

9 Feihl F., Liaudet L., Levy B.I., Waeber B. Hypertension and microvascular remodelling. Cardiovasc. Res. (2008) 78274 285.

10 Freitas F., Estato V., Carvalho V.F., Torres R.C., Lessa M.A., Tibirica E. Cardiac microvascular rarefaction in hyperthyroidism-induced left ventricle dysfunction. Microcirculation (2013) 20 590-598.

11 Su L., Dai Y., Deng W., Yin Y.H. Renin-angiotensin system blocking agents reverse the myocardial hypertrophy in experimental hyperthyroid cardiomyopathy via altering intracellular calcium handling. Zhonghua Xin Xue Guan Bing Za Zhi (2008) 36 744-749.

12 Kim B., Carvalho-Bianco S.D., Larsen P.R. Thyroid hormone and adrenergic signaling in the heart. Arq. Bras. Endocrinol. Metabol. (2004) 48 171-175.

13 Carvalho-Bianco S.D., Kim B.W., Zhang J.X. et al. Chronic cardiac-specific thyrotoxicosis increases myocardial betaadrenergic responsiveness. Mol. Endocrinol. (2004) 181840 1849.

14 Barreto-Chaves M.L., Carrillo-Sepulveda M.A., CarneiroRamos M.S., Gomes D.A., Diniz G.P. The crosstalk between thyroid hormones and the renin-angiotensin system. Vascul. Pharmacol. (2010) 52 166-170.

15 Klein I. Thyroid hormone and the cardiovascular system. Am. J. Med. (1990) 88 631-637.

16 Wu X.D., Dai D.Z., Zhang Q.P., Gao F. Propranolol and verapamil inhibit mrna expression of ryr 2 and serca in lthyroxin-induced rat ventricular hypertrophy. Acta Pharmacol. Sin. (2004) 25 347-351.

17 Kumamoto H., Okamoto H., Watanabe M. et al. Beneficial effect of myocardial angiogenesis on cardiac remodeling process by amlodipine and mci-154. Am. J. Physiol. (1999) 276 H1117-H1123.

18 Kobayashi N., Kobayashi K., Hara K. et al. Benidipine stimulates nitric oxide synthase and improves coronary circulation in hypertensive rats. Am. J. Hypertens. (1999) 12 483-491.

19 Rakusan K., Cicutti N., Kazda S., Turek Z. Effect of nifedipine on coronary capillary geometry in normotensive and hypertensive rats. Hypertension (1994) 24 205-211.

20 Dusseau J., Hutchins P.M. Calcium entry blockers stimulate vasoproliferation on the chick chorioallantoic membrane. Int. J. Microcirc. Clin. Exp. (1993) 13 219-231.

21 Asahi T., Shimabukuro M., Oshiro Y., Yoshida H., Takasu N. Cilazapril prevents cardiac hypertrophy and postischemic myocardial dysfunction in hyperthyroid rats. Thyroid (2001) 11 1009-1015.

22 Picard M.H., Adams D., Bierig S.M. et al. American society of echocardiography recommendations for quality echocardiography laboratory operations. J. Am. Soc. Echocardiogr. (2011) 24 1-10.

23 Basset A., Blanc J., Messas E., Hagege A., Elghozi J.L. Reninangiotensin system contribution to cardiac hypertrophy in experimental hyperthyroidism: an echocardiographic study. J. Cardiovasc. Pharmacol. (2001) 37 163-172.

24 Toyoda N., Yasuzawa-Amano S., Nomura E. et al. Thyroid hormone activation in vascular smooth muscle cells is negatively regulated by glucocorticoid. Thyroid (2009) 19 755-763.

25 Garcia S.I., Alvarez A.L., Porto P.I., Garfunkel V.M., Finkielman S., Pirola C.J. Antisense inhibition of 
thyrotropin-releasing hormone reduces arterial blood pressure in spontaneously hypertensive rats. Hypertension (2001) 37 365-370.

26 Sabino B., Lessa M.A., Nascimento A.R. et al. Effects of antihypertensive drugs on capillary rarefaction in spontaneously hypertensive rats: intravital microscopy and histologic analysis. J. Cardiovasc. Pharmacol. (2008) 51 402-409.

27 Mattfeldt T., Mall G., Gharehbaghi H., Moller P. Estimation of surface area and length with the orientator. J. Microsc. (1990) 159 301-317.

28 Estato V., Obadia N., Carvalho-Tavares J. et al. Blockade of the renin-angiotensin system improves cerebral microcirculatory perfusion in diabetic hypertensive rats. Microvasc. Res. (2013) 87 41-49.

29 Nascimento A.R., Machado M., de Jesus N. et al. Structural and functional microvascular alterations in a rat model of metabolic syndrome induced by a high-fat diet. Obesity (Silver Spring) (2013) 21 2046-2054.

30 Francischetti E.A., Tibirica E., da Silva E.G., Rodrigues E., Celoria B.M., de Abreu V.G. Skin capillary density and microvascular reactivity in obese subjects with and without metabolic syndrome. Microvasc. Res. (2011) 81 325-330.

31 Nascimento A.R., Lessa M.A., Sabino B., Bousquet P., Tibirica E. Microvascular effects of centrally acting antihypertensive drugs in spontaneously hypertensive rats. J. Cardiovasc. Pharmacol. (2010) 55 240-247.

32 Feihl F., Liaudet L., Waeber B., Levy B.I. Hypertension: a disease of the microcirculation? Hypertension (2006) 48 1012-1017.

33 Dong Z., Zeitlin B.D., Song W. et al. Level of endothelial cell apoptosis required for a significant decrease in microvessel density. Exp. Cell Res. (2007) 313 3645-3657.

34 de Jongh R.T., Serne E.H., Ijzerman R.G., de Vries G., Stehouwer C.D. Impaired microvascular function in obesity: implications for obesity-associated microangiopathy, hypertension, and insulin resistance. Circulation (2004) 109 2529-2535.

35 Paravicini T.M., Touyz R.M. Nadph oxidases, reactive oxygen species, and hypertension: clinical implications and therapeutic possibilities. Diabetes Care (2008) 31(Suppl 2) S170-S180.
36 Debbabi H., Uzan L., Mourad J.J., Safar M., Levy B.I., Tibirica E. Increased skin capillary density in treated essential hypertensive patients. Am. J. Hypertens. (2006) 19 477-483.

37 Antonios T.F. Microvascular rarefaction in hypertensionreversal or over-correction by treatment? Am. J. Hypertens. (2006) 19 484-485.

38 Hu L.W., Benvenuti L.A., Liberti E.A., Carneiro-Ramos M.S., Barreto-Chaves M.L. Thyroxine-induced cardiac hypertrophy: influence of adrenergic nervous system versus reninangiotensin system on myocyte remodeling. Am. J. Physiol. Regul. Integr. Comp. Physiol. (2003) 285 R1473-R1480.

39 Spoladore R., Fragasso G., Perseghin G. et al. Beneficial effects of beta-blockers on left ventricular function and cellular energy reserve in patients with heart failure. Fundam. Clin. Pharmacol. (2013) 27 455-464.

40 Takebayashi S., Li Y., Kaku T. et al. Remodeling excitationcontraction coupling of hypertrophied ventricular myocytes is dependent on t-type calcium channels expression. Biochem. Biophys. Res. Commun. (2006) 345 766-773.

41 Izumi T., Kihara Y., Sarai N. et al. Reinduction of t-type calcium channels by endothelin-1 in failing hearts in vivo and in adult rat ventricular myocytes in vitro. Circulation (2003) 108 2530-2535.

42 Meirelles Pereira L.M., Mandarim-de-Lacerda C.A. Effect of antihypertensive drugs on the myocardial microvessels in rats with nitric oxide blockade. Pathol. Res. Pract. (2000) 196 305-311.

43 Common K., Milburn K.S., Cawood T.S., Crozier I. Coronary artery spasm due to thyrotoxicosis. N. Z. Med. J. (2013) 126 75-78.

44 Canpolat U., Sunman H., Gurses K.M., Aytemir K. Vasospastic angina in a patient with hyperthyroidism. Herz (2012) 37 $570-572$.

45 Kim H.J., Jung T.S., Hahm J.R. et al. Thyrotoxicosis-induced acute myocardial infarction due to painless thyroiditis. Thyroid (2011) 21 1149-1151.

46 Al Jaber J., Haque S., Noor H., Ibrahim B., Al Suwaidi J. Thyrotoxicosis and coronary artery spasm: case report and review of the literature. Angiology (2010) 61 807-812.

47 Patel R., Peterson G., Rohatgi A. et al. Hyperthyroidismassociated coronary vasospasm with myocardial infarction and subsequent euthyroid angina. Thyroid (2008) 18 273-276. 